\title{
MANAGING CONTAINER RESHUFFLING IN VESSEL LOADING BY SIMULATION
}

\author{
Pasquale Legato \\ Rina Mary Mazza \\ Department of Informatics, Modeling, Electronics and System Engineering \\ University of Calabria \\ Rende, CS 87036, ITALY
}

\begin{abstract}
Planning the loading operations on a vessel in a container terminal is a very time-consuming activity. On one hand, the placement of the containers within the vessel must satisfy various constraints; on the other, reshuffles, i.e., unfruitful movements performed on the yard to retrieve a target container when it is not located on top of a stack, should be avoided. To support the work of terminal planners, we propose a simulation model that returns an accurate estimate of the number of reshuffles required with respect to a given loading sequence plan and a given yard configuration. The simulation model accounts for great details on operations under stochastic conditions. The estimates returned do not deteriorate as the dimension and complexity of the problem grow. Numerical experiments carried out for a real container terminal show how the model may easily support the planners in their daily job
\end{abstract}

\section{INTRODUCTION}

Container trade has grown significantly in the last few decades to represent approximately $17 \%$ of world seaborne trade by volume and $52 \%$ by value (United Nations 2012). With the aim of increasing their market share of containerized cargo within this pattern of trade, many container terminals have undertaken operational development programs focusing on organization, strategy, structure, processes, related internal rules and infrastructural conditions. In the medium term, the implementation of these programs is mostly based on the introduction of lean principles, which are expected to deliver efficient performance on several fronts, and lean tools, which are mean to support the port management in estimating the performance metrics of interest.

In (op. cit.), the UNCTAD reports on the collection and use of a set of performance indicators concerning both operational and financial aspects of port operation. However, operational indicators are regarded as of more direct concern to port management and, in particular, vessel turn-around time is considered an excellent operational indicator (United Nations 1967). It includes the time for vessel berthing, discharge, loading and departure, but the major component of the time measured by this indicator is represented by the vessel discharge and loading $(\mathrm{D} / \mathrm{L})$ process. Bearing in mind that the very large vessels of today carry thousands of containers, $\mathrm{D} / \mathrm{L}$ operations must be performed even more rapidly and efficiently.

In this paper we focus on the vessel loading process and, in particular, on the so-called loading sequence problem. In brief, when retrieving containers from the terminal yard to be loaded onto a vessel, one or more of the target containers may be stacked beneath other containers that are meant to be loaded on the same vessel or on a different one, but at a later time. So, in order to feed the loading process, container reshuffling (a.k.a. rehandling) must be performed, meaning that the containers stacked on top of the target containers must be first removed to reach the target containers and then repositioned. 
As a result, container reshuffling unavoidably affects the vessel turn-around time due to a loading service that forces the handling equipment on the yard to perform unproductive container moves.

The object of this study is to model and simulate the vessel loading process in order to support the terminal planners when they are called to provide an accurate estimate of the number of container reshuffling operations required. Although various mathematical programming models have been formulated with this purpose, the simulation model proposed is noteworthy in that i) it allows to represent the loading process in great detail and ii) it can estimate the number of container reshuffles required, whereas other approaches return approximated solutions that deteriorate as the dimension and complexity of the problem grow. The rest of this paper is organized as follows. The problem description and previous work are discussed in Section 2. A description of the simulation model is given in Section 3, followed by the presentation of numerical experiments in Section 4. Conclusions are finally drawn in Section 5.

\section{STOWAGE PLANNING}

In order to address the loading sequence problem, let us first introduce the concept of stowage and then discuss how a correct stowage planning affects the performance of both the container terminal and the container vessel.

The basic idea behind container stowage consists in providing the optimum placement of containers within a container vessel so that all constraints (restrictions placed upon where and how containers can be stowed) are satisfied and material handling costs (the costs associated with discharge, loading and transfer) are minimized, as in Wilson and Roach (1999). Since stowage activities are performed in connection with the vessel discharge and loading process, the proper arrangement of containers within both the container vessel and the container terminal can contribute to reducing the vessel turn-around time. Specifically, container stowage can become very time consuming because during container retrieval it is often necessary to reshuffle or rehandle some containers in order to gain access to those underneath. Container rehandling can either be required $a$ ) on board, because containers scheduled for discharge at port $\mathrm{x}$ are stacked beneath containers to be discharged at port $\mathrm{y}$ and the vessel calls port $\mathrm{x}$ before port $\mathrm{y}$, or $b$ ) on the yard, because containers scheduled for loading at the terminal are stacked on the yard below others which are to be loaded at a later time. The former case is of greater concern for the shipping line, whereas the terminal is more concerned with the second. In the literature, authors have addressed the ship stowage planning problem according to both perspectives, as discussed in the following sub-section.

\subsection{Literature Review}

According to the shipping line's point of view, Wilson (1997) and Wilson and Roach (1999) approach the problem with the aim of minimizing the material handling cost, while satisfying constraints on ship stability and other special storage requirements. Rather than just relying upon powerful computers to develop stowage solutions in which important problem features are left out in an attempt to reduce the problem to a scale that is solvable, research effort is placed on mapping the work of human planners and the content of the related documents into two different conceptual levels. Thus, the problem is decomposed into two sub-processes: a strategic planning process, in which "classes" of containers (i.e., containers sharing the same characteristics) are assigned to a blocked vessel space, and a tactical planning process, in which specific containers are assigned to specific vessel slots. The resulting hybrid approach adopted for problem solution is based on a variety of search algorithms (e.g., branch-and-bound, packing heuristics, and tabu search). In Avriel et al. (1998) the authors disregard vessel stability and several other constraints to focus on stowage planning. They propose a 0-1 binary linear programming formulation with the aim of minimizing the number of on-board rehandles. The optimal solution can be found for a single bay of a ship calling at a given number of ports, assuming that the number of containers to ship, along with their origin and destination ports, are known in advance. They present the so-called suspensory heuristic procedure, to improve the whole column heuristic procedure proposed in Avriel and Penn (1993) and based on binary linear programming as well, but still too large to be solved. Dubrovsky, Levitin and Penn 
(2002) propose a genetic algorithm based approach to solve the basic version of the problem. The authors give special care to the design of the algorithm to obtain a compact and efficient encoding, enable parallel implementation and allow flexibility in adding constraints (e.g., stability constraints that allow to keep the tilt below the dangerous threshold level during all legs of the voyage). In Ambrosino, Sciomachen and Tanfani (2006) the authors present a three-phase algorithm for defining stowage plans with the aim of minimizing the total loading time of the containers on the vessel satisfying weight, size, and stability constraints related to weight distribution. In the first phase the vessel is split into different portions and containers are associated to different subsets of bays without specifying their actual position. In the second stage, a $0 / 1$ linear programming model is solved with the aim of finding the optimal way for stowing containers in each partition of the vessel. In the last stage, possible unfeasibilities of the global solution due to the cross and horizontal stability of the vessel are checked and removed by performing local search exchanges. In Sciomachen and Tanfani (2007) the authors present a three-dimensional bin packing problem approach to minimize the total loading time of the stowage planning process and maximize the terminal's effective and mean net crane productivity. A three-stage procedure is proposed (i.e., bay partitioning and quay crane assignment, definition of the bins, and definition of the loading pattern), assuming that only standard containers are stowed, the number of containers to load on board is not greater than the number of available locations, the ship starts its journey in the port for which the problem is studied and successively visits a given number of other ports where only unloading operations are allowed. In Meisel and Wichmann (2010) the authors reshuffle containers directly within the bay of a vessel without temporarily unloading them. They present an optimization model that enables to exploit both the potential of quay crane double cycling (i.e., alternating loading and unloading operations), as well as the potential of internal reshuffles. The objective consists in finding a feasible sequence of container moves that converts a given arrival configuration of a bay into a given departure configuration within minimum service time. To solve problem instances of practical size, a multi-start meta-heuristic called greedy randomized adaptive search procedure (GRASP) is proposed.

More recently, various authors have also addressed the ship stowage planning problem from the terminal's point of view. Kim, Kang and Ryu (2004) present a beam search algorithm for the load sequencing of outbound containers in port container terminals with the aim of maximizing transfer crane (TC) and quay crane (QC) efficiency, while satisfying various constraints on stacking containers onto vessels. The load sequencing problem consists in two sub-problems: in the first problem, a pickup schedule is constructed in which the travel route of a transfer crane, as well as the number of containers it must pick up at each yard-bay are determined; in the second, the load sequence for individual containers is determined. The authors consider many practical constraints and objective functions (e.g., travel distance of TCs, handling convenience of TCs and QCs, maximum height of a stack in hold, the maximum total weight of containers on a hatch cover, the conformity of weights of loaded containers to the weight class specified in a stowage plan) and, above all, they are the first to simultaneously account for the load sequence of slots in a vessel and containers in a marshalling yard. In Alvarez (2006) a mixed integer formulation is presented to address a simplified version of the ship planning problem that accounts for container rehandling, distance traveled on the ground by the transport vehicles and vertical instability, while only one crew is assigned to the vessel. This formulation is used to provide a benchmark against a tabu search based algorithm for problem solution. The latter approach establishes which sections of the yard could provide containers of the needed type and generates a feasible tour of the yard. The tour specifies the order in which the yard section will be visited and the number of containers that will be collected from each section by the transfer cranes and transported towards the quay area by means of reach-stackers. In Imai et al. (2006) the authors define the ship stowage problem as an integer mathematical programming model with a single objective function that incorporates multiple objectives: three factors to account for ship stability (i.e., GM, list and trim) and one to estimate container rehandles. The novelty of their work consists in introducing the idea of probability in the mathematical programming model to estimate the number of rehandles. The solution procedure proposed uses a genetic algorithm. Monaco and Sammarra (2008) de- 
rives a linear integer model for the problem with the aim of minimizing container transportation time from the yard to the quay, along with the time needed to perform possible yard rehandles. The proposed formulation does not account for container rehandling on the same yard stack performed by different cranes. Feasible solutions to the problem are provided by a two-stage heuristic algorithm for solution construction and improvement.

\subsection{Managing Container Rehandles}

In this paper we embrace the terminal's point of view and focus on vessel loading within the ship stowage planning problem. In particular, we consider the so-called loading sequence problem with the aim of estimating the number or container rehandles to be performed on the yard with respect to the loading sequence determined by the terminal planners. Details pertaining to other aspects of the ship stowage planning problem (e.g., satisfaction of stability constraints) will not be provided because beyond the scope of this paper.

Estimating container rehandling on the yard is based on the input of three documents. The first is known as the prestow plan. It is provided by the planners of the shipping line and it specifies for each vessel slot the port of destination, size (e.g., standard, 45-footer, high-cube, oversized), type (e.g., reefer, dangerous, open-top) and weight (e.g., light, medium, heavy) of the container that can be stowed in that location. Containers bearing the same port of destination, size and type are said to belong to the same class. An example of a prestow plan for container loading alone is given in Figure 1 in which 7 containers are scheduled to be loaded: three are high-cube, 4 are reefer and all of them will be shipped to Algeciras (port of destination).

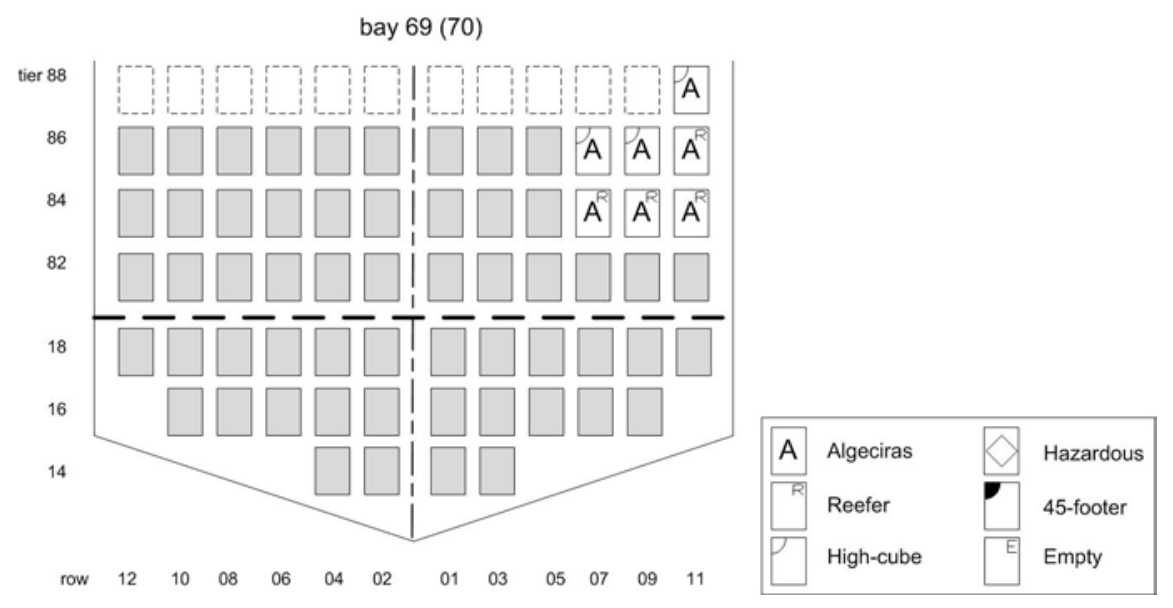

Figure 1: Example of a prestow plan.

According to the information contained in the prestow plan and once how many and which quay cranes will be assigned to discharge/load the vessel bays has been determined, the terminal planners construct a work schedule for each of the above quay cranes. The schedule normally specifies:

- the order of the bays to work on - even-numbered bays are for 40-foot locations, odd-numbered are for 20-foot locations;

- if discharge (D) or loading (L) operations are to be performed;

- if they are to take place on deck (D) or in hold $(\mathrm{H})$ - they are separated by hatchcovers which are represented in Figure 1 by the thick-dotted horizontal line;

- how many units are to be discharged/loaded for each type of container; 
- the number of moves left to go while operations are in progress.

An example of a work schedule for the loading operations to be performed by quay crane $\mathrm{n}^{\circ} 16$ assigned to rows 07-11 of bay 69 is illustrated in Figure 2.

Given the above documents, when drawing the third and last document called loading sequence plan terminal planners have "some" degrees of freedom in deciding exactly which container will be stowed into a given vessel slot.

\begin{tabular}{|c|c|c|c|c|c|c|c|c|c|c|c|c|c|c|}
\hline \multicolumn{4}{|c|}{ Visit: 391286} & \multicolumn{4}{|c|}{ Ship ID: ALBA VARDEN } & \multicolumn{5}{|c|}{ Planner: Franco Rossi } & \multicolumn{2}{|c|}{ Date: $15 / 02$} \\
\hline \multicolumn{2}{|c|}{ Crane: $\mathrm{C} 16$} & & & & & & & & & & \multicolumn{4}{|c|}{ Total Units: 7} \\
\hline Bay & $\mathrm{L} / \mathrm{D}$ & $\mathrm{D} / \mathrm{H}$ & 5 & 5 & 'F & $20^{\prime} \mathrm{E}$ & $40^{\prime} \mathrm{F}$ & $40^{\prime}$ & & & $\mathrm{RF}$ & OOG & EXP & To go \\
\hline 07 & L & I & & & 2 & & & & & 2 & 1 & & & 7 \\
\hline 09 & L & I & & & 2 & & & & & 2 & 1 & & & 5 \\
\hline 11 & $\mathrm{~L}$ & D & & & 3 & & & & & 3 & 2 & & & 3 \\
\hline & & & & & & & & & & & & & & \\
\hline & & & & & & & & & & & & & & \\
\hline & & & & & & & & & & & & & & \\
\hline
\end{tabular}

\begin{tabular}{|l|r|r|r|r|r|r|r|r|}
\hline Discharge & & & 0 & & 0 & & & 0 \\
\hline Load & & & 7 & & 0 & & & 7 \\
\hline Restow & & & & & & & & \\
\hline Total & & & 7 & & 0 & & & 7 \\
\hline
\end{tabular}

Figure 2: Example of a work schedule for a given quay crane.

For instance, according to the pre-stow plan provided by the shipping line and illustrated by plan (a) in Figure 3, when operating according to a sea-to-shore loading pattern, the crane driver must first load container 2 and then container 1 in order to remain compliant to the related security issues.
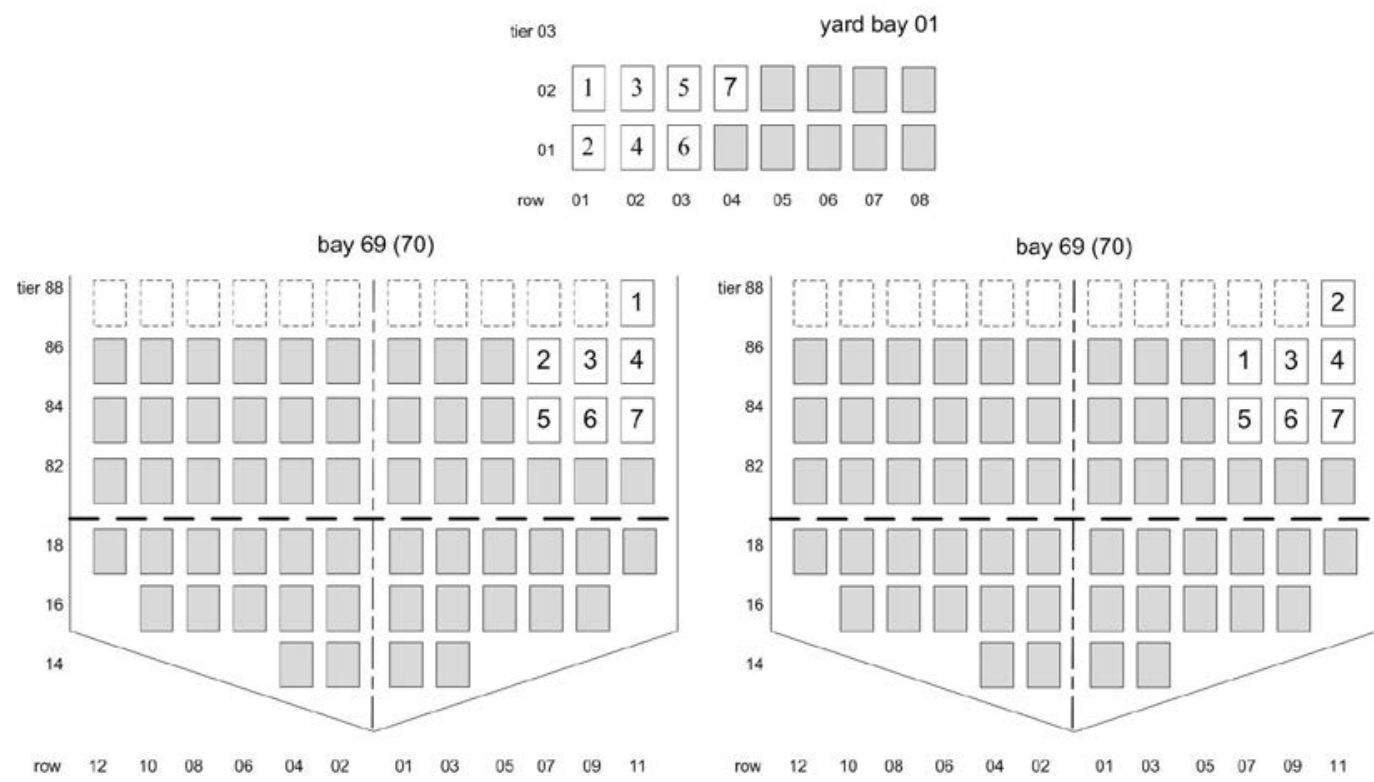

plan (a)

plan (b)

Figure 3: Alternative loading sequences bearing a different number of containers to be rehandled. 
This specific order causes an unfruitful container movement in the yard $\left(\mathrm{n}^{\circ}\right.$ reshuffles $\left.=1\right)$. However, if containers 1 and 2 belong to the same class, their location on the vessel can be exchanged as illustrated by plan (b) in Figure 3, provided that the maximum stack weights are preserved. The loading sequence plan for the example provided is illustrated in Figure 4. Thus, the terminal planner can build an optimized loading sequence plan in which the internal and unfruitful movements are reduced ( ${ }^{\circ}$ reshuffles $\left.=0\right)$.

\begin{tabular}{|r|r|r|r|r|}
\hline $\mathrm{QC} \mathrm{n}^{\circ}$ & Sequence & Container $^{\circ}$ & Yard location & Vessel location \\
\hline 16 & 1 & 5 & $(01,03,02)$ & $(69,07,84)$ \\
\hline 16 & 2 & 1 & $(01,01,02)$ & $(69,07,86)$ \\
\hline 16 & 3 & 6 & $(01,03,01)$ & $(69,07,84)$ \\
\hline 16 & 4 & 3 & $(01,02,02)$ & $(69,09,86)$ \\
\hline 16 & 5 & 7 & $(01,04,02)$ & $(69,11,84)$ \\
\hline 16 & 6 & 4 & $(01,02,01)$ & $(69,11,86)$ \\
\hline 16 & 7 & 2 & $(01,01,01)$ & $(69,11,88)$ \\
\hline
\end{tabular}

Figure 4: Example of a loading sequence plan.

At this point, one may observe that the planning of a container vessel is an off-line optimization problem, while counting the yard reshuffles can only be done during the loading process. As well put by Imai et al. (2006), when loading containers the loading sequence is predetermined and this implies that container retrieval is programmed and not random. Consequently, in principle, the number of rehandles can be calculated exactly. In practice, the exact calculation is based on the respect of the predetermined loading sequence which features both random events and activity durations. So, due to this nature of the problem, it is quite difficult or even impossible to formulate the problem as a mathematical programming model, with evaluation of the exact number of rehandles. Exceptions can only be found for very simple cases, for instance, in which a container vessel is loaded by one crane and container transfer between the yard and quay is performed by only one transportation vehicle.

In most of the cases, one must resort to simulation. The simulation model presented in the next section is meant to support the terminal planners when they are called to estimate the number of rehandles associated with a specific loading sequencing plan, rather than just leaving them with viable rules of the thumb.

\section{SIMULATION MODEL}

Starting from the general information provided in the previous section, the loading sequence process is now tailored to mirror the organization and infrastructures of the transshipment container terminal located at the port in Gioia Tauro, Italy. In this facility, to perform loading operations, rail-mounted cranes in the quay area retrieve containers from a limited buffer area at the feet of the cranes where containers are left by straddle carriers (SCs) - very flexible and productive container equipment used to lift and transfer containers at high speed. These basic characteristics are accounted for in the step-by-step representation or conceptual model given in Figure 5 in which the containers flow through the model and, according to the on-going activities, request, seize and release both active (i.e., SCs and quay cranes) and passive resources (i.e., buffer space under the quay cranes and rows in the yard blocks). The overall description of the model is provided in the following.

A container listed in the loading sequence plan requests an SC to be retrieved from the yard. If the SC is not available, the container must wait; otherwise, the SC starts to travel towards the corresponding yard block. Once on target, the SC must gain access to the row in which the container is stacked. For security reasons, if other SCs are already performing operations in the same row or in adjacent ones, the SC must queue in front of the row and wait for its turn according to a FIFO logic. 


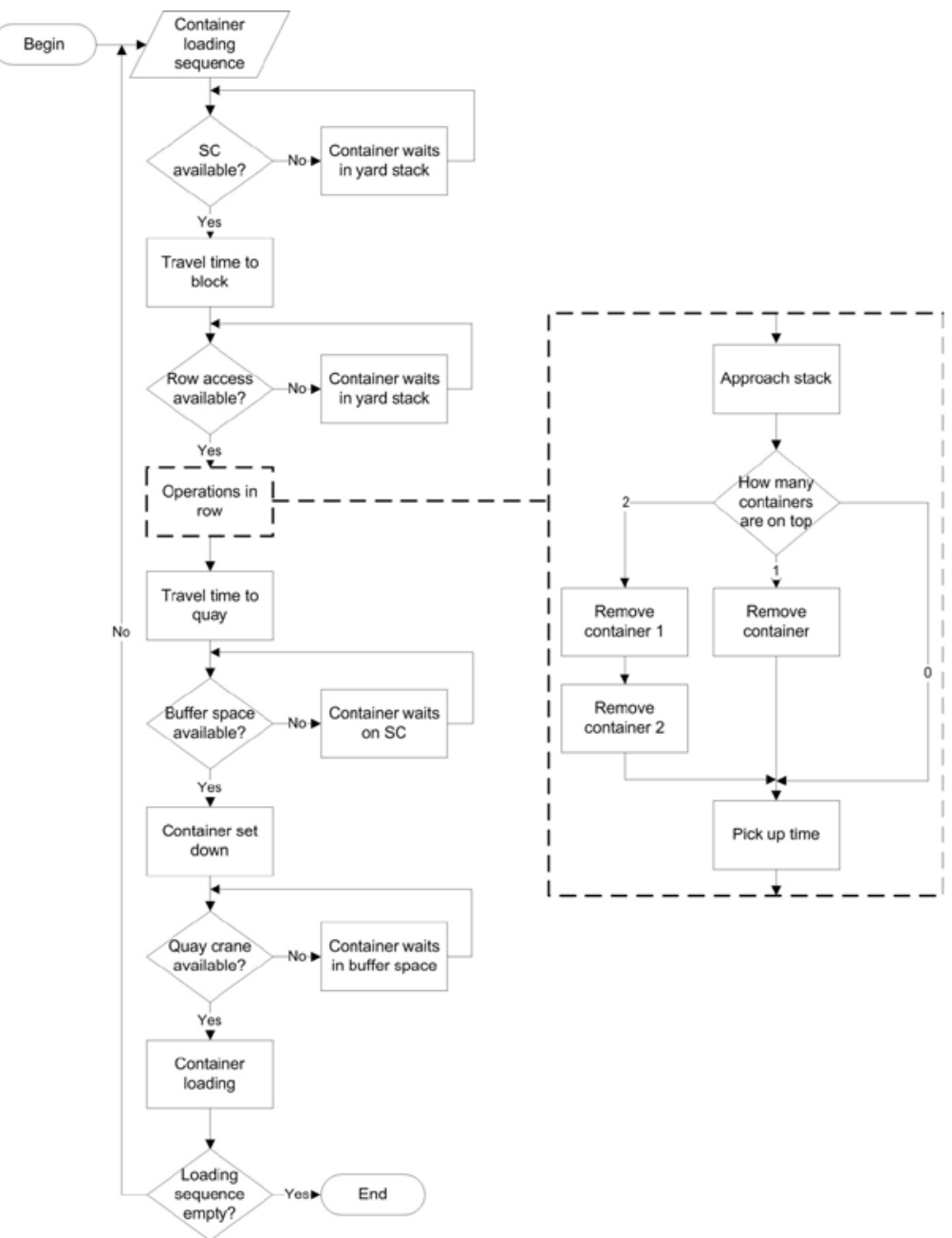

Figure 5: Step-by-step description of the loading sequence process.

After gaining access to the row, operations take place, as illustrated by the zoom in Figure 5. The operation time includes the time to $i$ ) approach the stack, $i$ ) remove and reallocate other containers eventually located on top of the target container and iii) then, finally, pick up the container. The container is now transferred towards the quay where it is either set down by the $\mathrm{SC}$ at the feet of the quay crane if buffer space is available or left waiting on the SC for buffer space to become available. Once set down, the container is loaded by the quay crane according to the schedule of the loading sequence plan.

Besides the limited number of resources that can be deployed to carry out the loading sequence and the operational restrictions imposed by security measures, the simulation model also features non deterministic crane service times and SC service times, as well as a highly-detailed container flow between the bordering areas of the terminal which is affected by:

- the travelling status of the SCs (with or without containers);

- the number of reshuffles to be performed to reach a stacked container and, as a result, the number of SCs queuing before a row of a yard block; 
- the availability of buffer space under the crane and, as a result, the number of SCs queuing before the crane buffer.

So, in addition to estimating the number of reshuffles to be performed on the yard, model solution via simulation also supports terminal planners in the evaluation of alternative loading sequences by providing them with further insight on crane starvation (i.e., too little SCs), block before service (i.e., too many $\mathrm{SCs}$ ) and, if required, other unexpected occurrences (technical breakdowns, maintenance, environmental interruptions, etc.)

Technically speaking, the simulation model is developed according to an event-scheduling world view (Banks et al. 2001). The events occurring within the simulation model are listed in Table 1.

Table 1: Discrete events of the simulation model.

\begin{tabular}{|l|l|c|c|}
\hline \multirow{2}{*}{ Event } & \multicolumn{2}{c|}{ Activities } & \multicolumn{2}{c|}{ Resources } \\
\cline { 3 - 4 } & & Seize & Release \\
\hline SCtraveltoyard & schedules containerpickup or queues request & SC & \\
\hline Containerpickup & schedules SCtravelfromyard or queues request & row yard & \\
\hline SCtravelfromyard & schedules containersetdown & & row yard \\
\hline containersetdown & schedules containerload or queues request & buffer space & \\
\hline Containerload & schedules containeronboard or queues request & crane & SC \\
\hline containeronboard & schedules no actions & & $\begin{array}{c}\text { buffer space } \\
\text { crane }\end{array}$ \\
\hline
\end{tabular}

Every event of the list calls for the scheduling of specific activities and, thus, triggers other events. With respect to the main objective of the model, the containerpickup event is of particular interest because the activities it calls are those that may determine an increase in the number of reshuffles to be performed on the yard.

\section{NUMERICAL EXPERIMENTS}

The numerical experiments presented in this section show how the simulator is used by terminal planners to estimate the number of rehandles associated with a specific loading sequencing plan.

The simulator is developed in Microsoft Visual Basic 6.0 Professional and experiments are all run on a personal computer equipped with a $2.26 \mathrm{~Hz}$ Inter Core ${ }^{\mathrm{TM}} 2$ duo processor and $2.93 \mathrm{~GB}$ of RAM. The problem input and the simulation parameters are specified via the GUI panel illustrated in Figure 6. In particular, the loading sequence and the yard configuration are brought in via Excel. For every quay crane assigned to the vessel, the loading sequence file contains a sheet in which containers are listed according to the scheduled order, along with their (bay, row, tier) coordinates and the distance between the yard location of each container and the corresponding loading point on the departing vessel. The yard configuration file uses the same coordinates to represent the status of the entire yard which, in turn, is dynamically updated while the container transfer operations towards the quay area are taking place. The crane and vehicle configurations are defined by entering the corresponding number of units deployed and their average service times. The experiment setup is completed by providing the seed and the number of simulation runs to be performed.

As required by any thorough and sound simulation study, a first set of ad hoc instances are used to verify and validate the simulation model. Verification is carried out by simulating predetermined loading sequences in which operations are performed by one quay crane and one SC. In these cases, given a yard configuration, the number of rehandles can be calculated exactly and then compared to the estimates returned by the simulator. For instance, given the yard configuration in Figure 7, one may easily verify by hand that in both cases the number of reshuffles estimated by the simulator (i.e., 0 and 8 , respectively) is correct. 


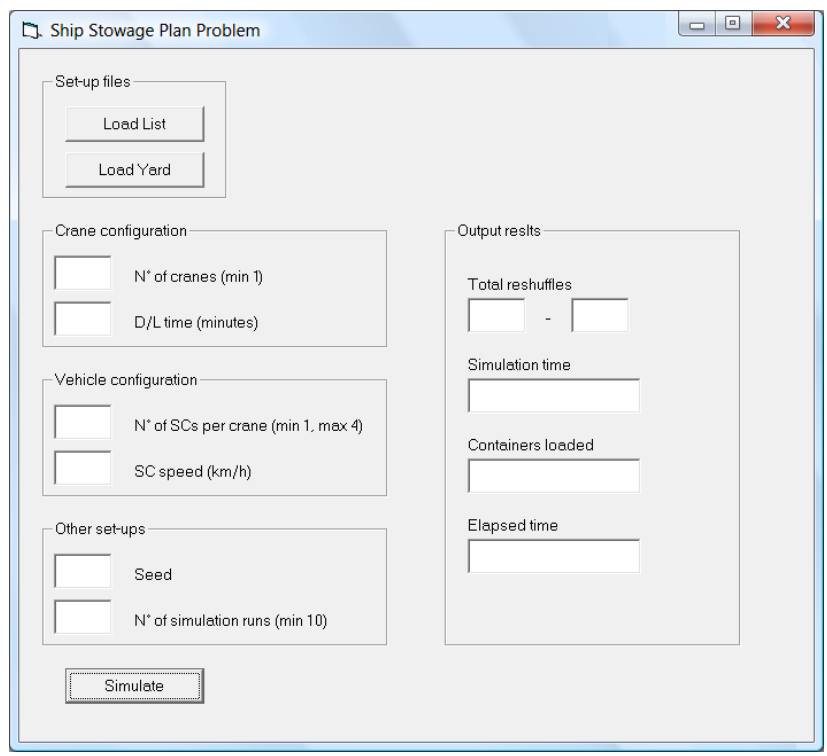

Figure 6: A snapshot of the ship stowage plan simulator.

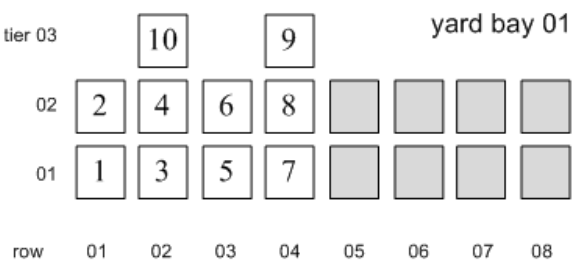

\begin{tabular}{|r|r|r|}
\hline Sequence & Container $\mathrm{n}^{\circ}$ & Yard location \\
\hline 1 & 2 & $(01,01,02)$ \\
\hline 2 & 1 & $(01,01,01)$ \\
\hline 3 & 10 & $(01,02,03)$ \\
\hline 4 & 4 & $(01,02,02)$ \\
\hline 5 & 3 & $(01,02,01)$ \\
\hline 6 & 6 & $(01,03,02)$ \\
\hline 7 & 5 & $(01,03,01)$ \\
\hline 8 & 9 & $(01,04,03)$ \\
\hline 9 & 8 & $(01,04,02)$ \\
\hline 10 & 7 & $(01,04,01)$ \\
\hline
\end{tabular}

\begin{tabular}{|c|c|c|}
\hline Sequence & Container $n^{\circ}$ & Yard location \\
\hline 1 & 1 & $(01,01,01)$ \\
\hline 2 & 2 & $(01,01,02)$ \\
\hline 3 & 3 & $(01,02,01)$ \\
\hline 4 & 4 & $(01,02,02)$ \\
\hline 5 & 5 & $(01,03,01)$ \\
\hline 6 & 6 & $(01,03,02)$ \\
\hline 7 & 7 & $(01,04,01)$ \\
\hline 8 & 8 & $(01,04,02)$ \\
\hline 9 & 9 & $(01,04,03)$ \\
\hline 10 & 10 & $(01,02,03)$ \\
\hline \multicolumn{3}{|c|}{ reshr } \\
\hline
\end{tabular}

Figure 7: Verifying the reshuffle estimates returned by the simulator for a simple yard configuration and two alternative loading sequences.

Validation is conducted on real loading sequences provided by Medcenter Container Terminal SpA, the company that runs the terminal in Gioia Tauro. In particular, by relaxing some of the major constraints on resource availability (i.e., a finite number of straddle carriers and a limited buffer space at the feet of the quay cranes) and operation rules (i.e., SCs may not simultaneously work on adjacent row 
yards), the simulator returns estimates that are consistent with those obtained by using the terminal planners' rules of the thumb.

In the second set of experiments, different size instances are built on real data and served as an input to the simulator with the aim of estimating the number of reshuffles generated by the related loading sequences. In particular, a so-called fixed-sample-size method is used to construct $95 \%$ confidence intervals for the number of reshuffles. Considering a sample size $n=50$, if the symmetric density or skewness of this performance measure is small, the coverage level of the confidence interval should hold to a good approximation, as explained in Nakayama (2006). This happens to be our case: the skewness of the distribution of the number of reshuffles decreases as the number of SCs deployed per quay crane grows, as shown by the example in Figure 8. This behavior could be explained by observing that, as the number of SCs grows, so does the probability that SCs working on the same yard block may either facilitate or impede the retrieval activities of other containers. Thus, values for the number of reshuffles falling on either side of the mode are, more or less, balanced.

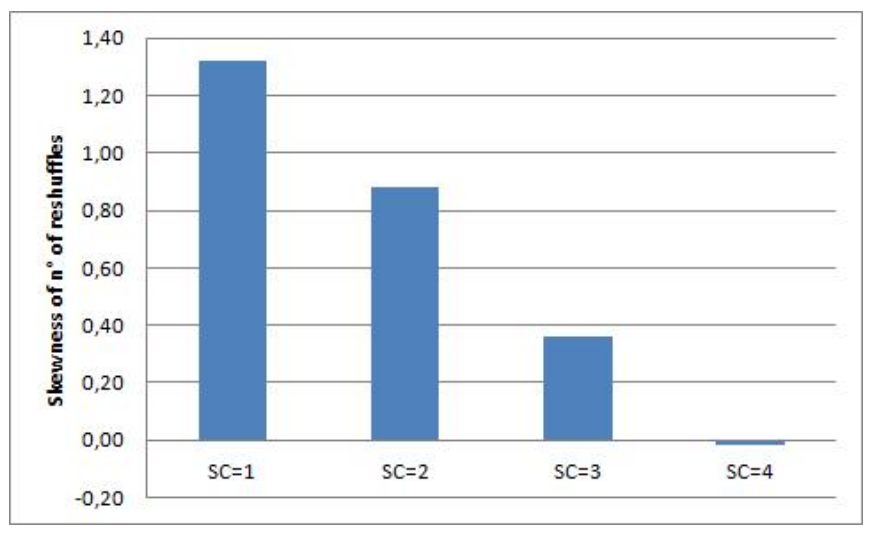

Figure 8: Example of how the skewness of the distribution of the number of reshuffles decreases as the number of SCs grows.

The interval estimates returned by the simulator are compared against an upper bound on the number of reshuffles which is obtained by applying a common rule of the thumb, i.e., considering how many reshuffles would be necessary if, for a given vessel, each single crane-SC couple were the only one to be performing container handling between the quay and yard areas.

This stated, let us consider three real loading plans: in the first, 84 containers are loaded by one quay crane onto a feeder vessel; in the second, 360 containers are loaded onto an oceanic vessel by two quay cranes; in the third, 633 containers are loaded onto an oceanic vessels by three quay cranes. The number of SCs assigned to a single crane usually ranges from 1 to 4 , according to the distance between the loading area and the container retrieval points on the yard. As for service times, according to company statistics, quay cranes load containers according to a 16-order Erlang distribution law with average value equal to two minutes, whereas SCs transfer containers at a constant rate of $15 \mathrm{kmph}$.

The results in the "Reshuffles" columns of Table 2 carry different types of information. According to the figures provided, with respect to a single loading sequence the estimated number of reshuffles does not vary across the four scenarios, each of which bears a different number of SCs. This is probably due to the fact that the number of SCs alone cannot affect yard traffic: they must also be considered in conjunction with the locking phenomena occurring on the single rows of the yard where other SCs are already working. The above columns then allow to compare the confidence intervals returned by the simulator (CI) with the upper bounds (UB) provided by the given rule of the thumb. As one may observe, results are substantially equivalent only for instance $n^{\circ} 1$, whatever be the number of SCs deployed. Instead, things 
are quite different for instances 2 and 3: UB values are greater than CI values by a factor of 3 (86 vs. 33) and 10 (209 vs 24), respectively. This particular outcome leads to a first conclusion: in general, simple rules of the thumb are likely to provide the terminal planner with an easy and fast approximation of the number of reshuffles, but, unfortunately, this approximation deteriorates as the problem dimensions grow. Hence, simulation may well play its role.

Table 2: Performance results for different size real-world loading sequences.

\begin{tabular}{|c|c|c|c|c|c|c|c|c|c|}
\hline & \multicolumn{3}{|c|}{84 containers, $1 \mathrm{QC}$} & \multicolumn{3}{|c|}{360 containers, 2 QCs } & \multicolumn{3}{|c|}{633 containers, 3 QCs } \\
\hline $\mathrm{SC}$ & Reshuff & & TLT (min) & Reshuffl & & TLT (min) & Reshuff & & TLT (min) \\
\hline $\mathrm{n}^{\circ}$ & $\mathrm{CI}$ & B & $\mathrm{CI}$ & $\mathrm{CI}$ & $\mathrm{IB}$ & $\mathrm{CI}$ & $\mathrm{CI}$ & UB & $\mathrm{CI}$ \\
\hline 1 & 18 & \multirow{4}{*}{18} & $1875-1901$ & $32.64-33.24$ & \multirow{4}{*}{86} & $4476-4512$ & $23.67-24.05$ & \multirow{4}{*}{209} & 6394-6873 \\
\hline 2 & $7.38-17.74$ & & 944-956 & $32.82-33.38$ & & $2246-2261$ & $23.71-24.25$ & & 3363 \\
\hline 3 & 17.02 .17 .38 & & $637-645$ & $33.27-34.05$ & & $1504-1517$ & $23.75-24.29$ & & $2249-2263$ \\
\hline 4 & $17.06-17.46$ & & $479-486$ & $33.08-34.12$ & & $1134-1144$ & $24.32-24.84$ & & $1695-1706$ \\
\hline
\end{tabular}

Table 2 also reports on the interval estimates returned by the simulator for the total loading time (TLT) required to carry out each loading sequence. Estimates for this performance measure (obviously) decrease as the number of SCs grows. This result leads to a second, yet equally important conclusion: even though the number of reshuffles does not vary, deploying multiple SCs during the vessel loading process is crucial with respect to minimizing the vessel turn-around time and other related performance measures such as gross crane productivity (i.e., the average number of container movements performed per hour by the assigned quay crane(s)) and utilization of the quay cranes.

\section{CONCLUSIONS}

We have successfully addressed the vessel loading process from a container terminal's point of view. We designed and implemented a user-friendly discrete-event simulator of the loading sequence problem with the aim of estimating the number of reshuffles to be performed on the yard with respect to a given loading plan and a given yard configuration. The simulation model proposed allowed to $i$ ) represent the loading process in great detail and $\mathrm{ii}$ ) estimate the number of container reshuffles required, whereas other approaches could only return approximated solutions that deteriorated as the dimension and complexity of the problem grew. Numerical experiments showed how the simulator could systematically support the planners of a real terminal in their daily job, rather than just leaving them to use viable rules of the thumb. These rules worked well for small loading plans but they fell far short - even by a factor of 10 - of providing accurate estimates of the number of reshuffles for larger size loading plans.

\section{REFERENCES}

Álvarez, J. F. 2006. "A Heuristic for Vessel Planning in a Reach Stacker Terminal” Journal of Maritime Research 3: 3-16.

Ambrosino, D., A. Sciomachen and E. Tanfani. 2006. "A Decomposition Heuristics for the Container Ship Stowage Problem.” Journal of Heuristics 12: 211-233.

Avriel, M. and M. Penn. 1993. "Exact and Approximate Solutions of the Container Ship Stowage Problem." Computers and Industrial Engineering 25:271-274.

Avriel, M., M. Penn, N. Shpirer, and S. Witteboon. 1998. "Stowage Planning for Container Ships to Reduce the Number of Ships." Annals of Operations Research 76:55-71.

Banks, J., J. S. Carson, B. L. Nelson, and D. M. Nicol. 2000. Discrete-Event System Simulation. 3rd ed. Upper Saddle River, New Jersey: Prentice-Hall, Inc.

Dubrovsky, O., G. Levitin and M. Penn. 2002. "A Genetic Algorithm with a Compact Solution Encoding for the Container Ship Stowage Problem.” Journal of Heuristics 8: 585-599. 
Imai, A., K. Sasaki, E. Nishimura, and S. Papadimitriou. 2006. "Multi-Objective Simultaneous Stowage and Load Planning for a Container Ship with Container Rehandle in Yard Stacks." European Journal of Operational Research 171: 373-389.

Kim, K. H., J. S. Kang and K. R. Ryu. 2004. "A Beam Search Algorithm for the Load Sequencing of Outbound Containers in Port Container Terminals." OR Spectrum 26: 93-116.

Meisel, F. and M. Wichmann. 2010. "Container Sequencing for Quay Cranes with Internal Reshuffles." OR Spectrum 32:569-591.

Monaco, M. F. and M. Sammarra. 2008. "The Ship Stowage Planning Problem.” In Proceedings of the 11th International Workshop on Harbor, Maritime \& Multimodal Logistics \& Simulation, Edited by A. Bruzzone, F. Longo, Y. Merkuriev, G. Mirabelli, and M. A. Piera, 208-213. Rende (CS), Italy.

Nakayama, M. 2006. "Output Analysis for Simulation.” In Proceedings of the 2006 Winter Simulation Conference, Edited by L. F. Perrone, F. P. Wieland, J. Liu, B. G. Lawson, D. M. Nicol, and R. M. Fujimoto, 36-46. Piscataway, New Jersey: Institute of Electrical and Electronics Engineers, Inc.

Sciomachen, A. and E. Tanfani. 2007. "A 3D-BPP Approach for Optimising Stowage Plans and Terminal Productivity.” European Journal of Operational Research 183: 1433-1446.

United Nations. 1967. "The Turn-Around Time of Ships in Port." New York and Geneva: United Nations.

United Nations. 2012. "Port Developments." In Review of Maritime Transport 2012, 80-94. New York and Geneva: United Nations.

Wilson, I. D. 1997. "The Application of Artificial Intelligence Techniques to the Deep-Sea ContainerShip Cargo Stowage Problem." Ph.D. thesis, Department of Industrial Engineering, North Carolina State University, Raleigh, North Carolina. School of Accounting and Mathematics, Division of Mathematics and Computing, The University of Glamorgan, Pontypridd, Wales, UK. http://intelligence.research.glam.ac.uk/documents/download/1/ [Accessed March 15, 2013].

Wilson, I. D. and P. A. Roach. 1999. "Principles of Combinatorial Optimization Applied to ContainerShip Stowage Planning." Journal of Heuristics 5:403-418.

\section{AUTHOR BIOGRAPHIES}

PASQUALE LEGATO is an Associate Professor of Operations Research in the Department of Informatics, Modeling, Electronics and System Engineering at the University of Calabria, Rende (CS, Italy), where he teaches courses on simulation for system performance evaluation. He has published on queuing network models for job shop and logistic systems, as well as on integer programming models. He has been involved in several national and international applied research projects and is serving as a reviewer for many international journals. His current research activities focus on the development and analysis of queuing network models for logistic systems, discrete-event simulation and the integration of simulation output analysis techniques with combinatorial optimization algorithms for real-life applications in transportation and logistics. His email is legato@deis.unical.it and his web page is www.deis.unical.it/legato.

RINA MARY MAZZA is the head of the "Research Projects Office" in the Department of Informatics, Modeling, Electronics and System Engineering at the University of Calabria, Rende (CS, Italy). She received a Ph.D. degree in Operations Research from the above university. She has a seven-year working experience on knowledge management and quality assurance in research centers. She is also a consultant for operations modeling and simulation in terminal containers. Her current research interests include discrete-event simulation and optimum-seeking by simulation in complex logistic systems. Her e-mail address is rmazza@deis.unical.it. 